\title{
THEORY OF INTRINSIC VARIABILITY IN HOT-STAR WINDS
}

\author{
Stanley P. Owocki \\ Bartol Research Institute \\ University of Delaware \\ Newark, DE 19716 USA
}

\begin{abstract}
The winds of the hot, luminous, O, B, and WR stars are driven by the linescattering of the star's continuum radiation flux. Several kinds of observational evidence indicate that such winds are highly structured and variable, and it seems likely that a root cause of this variability is the known strong instability of the line-driving mechanism. Initial dynamical models of the nonlinear evolution of this instability confirm that the wind indeed becomes highly structured, with large amplitude $(\sim 500-1000 \mathrm{~km} / \mathrm{s})$ shocks that separate high-speed rarefied flow from lower speed, dense shells. Remarkably, such variability can often have an intrinsic character, persisting even in the absence of explicit perturbation, and it now appears that this is a direct consequence of a degeneracy in the steady-state solutions for such models. However, recent work indicates that including scattering effects, which have so far been ignored in these pure-absorption models, might reduce or even break this steady-state solution degeneracy; through the "line-drag" effect, scattering can also reduce the strength of the instability, possibly rendering it an advective character for which wind variability now requires explicit perturbation from below. This review will examine the consequences of these ideas for understanding the likely nature of wind variability among the various kinds of early-type stars.
\end{abstract}

\section{Introduction}

Although this conference focuses on the properties of Wolf-Rayet stars and their winds, this review on the theory of intrinsic wind variability will deal primarily with the related $O$ and $B$ stars. The reason for this is that for the massive Wolf-Rayet winds there are still no completely satisfactory models to explain even the mean, time-averaged properties, e.g., the large mass loss rate, and so there is little solid basis on which to build a much more complicated picture of instability and variability. Nonetheless, it is generally thought that the basic driving mechanism for Wolf-Rayet winds is similar to that of OB stars, namely scattering of the star's strong radiation field in spectral lines of heavy minor ions in the wind (Lucy and Solomon 1970; Castor, Abbott, and Klein 1975, hereafter CAK). For these OB stars, steady-state wind theories have reached a quite high level of sophistication (Abbott 1980; Pauldrach, Puls, and Kudritzki 1986) and their predictions of gross wind properties like the time-averaged mass loss rate or terminal flow speed are in quite good agreement with values inferred by observation. (See, e.g., recent reviews by Abbott 1988, Kudritzki and Hummer 1990, and Owocki 1990.)

Despite this general success of steady-state models, there are numerous observational phenomena that seem to imply that such line-driven winds are not at all smooth, timesteady flows, but rather are both spatially and temporally highly variable. These include: soft X-ray emission (Harnden et al. 1979); nonthermal radio emission (Abbott, Bieging, and Churchwell 1984); line profile variations in UV spectra of OB stars ("narrow absorption 
components"; Lamers and Morton 1976; Prinja and Howarth 1988) or in optical spectra of WR stars (Robert and Moffat 1990); and blackness of saturated UV profiles from OB stars (Lucy 1982a).

It now seems likely that this observationally inferred variability is an intrinsic property of the wind for which a root cause is the inherent instability of the line-driving mechanism. This means that the wind variability does not require external driving by, say, large-amplitude pulsation of the underlying star. Of course, to the extent that such stars might in fact exhibit pulsations (Baade 1988), these would have a definite impact on any wind variability (Willson and Hill 1979; Castor 1987), but such a circumstance will not be a focus of this review. Rather, the emphasis here will be on the physics of line-driven mass loss, its fundamental instability, and how this can by itself give rise to intrinsic wind variability that persists even without explicit excitation from the underlying star.

\section{Linear Theory of the Line-Driven Instability}

The basic physics of this line-driven instability is quite simple (Lucy and Solomon 1970; see review by Rybicki 1987): a small-scale increase in radial flow speed Doppler-shifts the local line-frequency out of the absorption shadow of underlying material, leading to an increased radiative force which then tends to further increase the flow speed. However, even in the linear case of small amplitude perturbations, there are two important modifications to this simple picture. First, the perturbation must also be on a small enough scale so as to remain optically thin (MacGregor, Hartmann, and Raymond 1979; Carlberg 1980), which allows one to ignore any change in the absorption shadow itself. As shown by Owocki and Rybicki (1984), the limiting scale is roughly the Sobolev length $L \equiv v_{\text {th }} /(d v / d r)$, over which the mean wind velocity $v$ increases by an ion thermal speed $v_{\text {th }}$. Perturbations with a scale larger than this can be analyzed using the same Sobolev (1960) approximation methods that are used to model the mean outflow (Abbott 1980). The line-force is then proportional to the velocity gradient, and since this implies that the perturbed force is out of phase with the perturbed velocity, no net work can be done on such large-scale perturbations, which means they are stable.

The second modification to the above simple picture has to do with its neglect of the diffuse radiation that results from the nearly pure-scattering character of most driving lines. In a frame comoving with the mean flow, this diffuse radiation has a near fore-aft symmetry, implying that its associated net mean force can be neglected in favor of the much stronger direct absorption term. However, Lucy (1984) first pointed out that, as seen by a small-scale velocity perturbation $\delta v$, this diffuse field is stronger by a factor $\delta v / v_{\text {th }}$ against the direction of the perturbed velocity. As a result, there is a net drag force that is on the same order as the perturbed direct force that gives rise to the instability. Very near the star this can in fact completely eliminate the instability, but away from the wind base, the combined effects of spherical expansion and the shrinking of the solid angle subtended by the stellar core sharply reduce the relative importance of this line-drag effect. At $r=1.5 R_{*}$, for example, the net growth rate is about half that obtained from a pure-absorption analysis (Owocki and Rybicki 1984, 1985).

This still implies a strong instability, because the basic growth rate associated with the direct-absorption term is quite large, roughly $\Omega \approx g_{\mathrm{rad}} / 2 v_{\text {th }}$, i.e., about half the mean radiative acceleration rate through a thermal speed. Since $g_{\mathrm{rad}} \approx v(d v / d r)$, this can alternatively be written as $\Omega \approx v / 2 L$, i.e., about half the flow rate through a Sobolev length. If we compare this to a typical expansion rate $v / H$, where $H=v /(d v / d r)$, we see that the growth rate is a factor $H / 2 L=v / 2 v_{\text {th }}$ higher. In these highly supersonic winds, this implies on the order of $50-100$ e-folds of cumulative growth for a typical wind perturbation! In practice, of course, this means that any perturbation with a small but finite 
amplitude would quickly become nonlinear in the wind. The remaining sections review various attempts to determine the nature of the resulting nonlinear wind structure.

\section{Heuristic Models of a Wind with Embedded Shocks}

Given the supersonic nature of the wind outflow and the large instability growth rate, it seems inevitable that, whatever its detailed form, the nonlinear wind structure arising from this instability will include shocks. Several of the attempts to study the consequences of this instability have thus centered on determining the properties of these assumed shocks. (See review by Castor 1987).

The first models along these lines (e.g., Lucy 1982b) assumed the wind contains a quasi-periodic train of forward shocks. Relatively fast flow exposed to a nearly unshadowed stellar flux is strongly driven, and so is pushed against relatively slow flow, which is shadowed by the fast material and only weakly driven. A forward shock thus forms that sweeps up and accelerates this slower material, thereby adding it to the faster flow. A crucial point is that the fast material represents post-shock flow, with its associated high density and (at least initially) high temperature. To maintain the structure, this material must quickly cool and reform the driving ions that line-absorb the radiative momentum.

The question of the ionization and energy balance behind the shock was analyzed by Krolik and Raymond (1985) for a single, forward shock that propagates outward through the wind. For massive winds, they inferred that the cooling would be sufficiently rapid to allow the driving ions to reform and so maintain the structure. For low-density winds, however, they argue that the shock amplitude would be limited by the requirement that the cooling time be less than the flow time.

MacFarlane and Cassinelli (1989) studied the evolution of wind shocks with a phenomenological numerical-hydrodynamics model in which an initially smooth, slow wind is disrupted by a sudden increase in the driving force, thereby accelerating the wind to a much higher speed. For parameters chosen to give initial and final state terminal speeds of $500 \mathrm{~km} / \mathrm{s}$ and $2500 \mathrm{~km} / \mathrm{s}$, collision between the fast and slow flow forms a forward/reverse pair of shocks of velocity amplitude $\sim 1000 \mathrm{~km} / \mathrm{s}$ each. This turns out to be just what's needed to reproduce the observed X-ray properties of their model star, $\tau$ Sco.

\section{Radiation-Hydrodynamics Simulation of Non-Linear Structure}

Owocki, Castor, and Rybicki (1988; hereafter OCR) have recently developed a numerical, radiation-hydrodynamics code aimed at directly simulating the dynamical evolution of the line-driven wind instability, and thereby determining the likely nature of the resulting nonlinear wind structure. Because the instability occurs for perturbations with a length scale near and below the Sobolev length, OCR had to develop a method for computation of the line force which did not use the Sobolev approximation, but which still avoided the inordinate computational expense of solving the full line-transfer problem at each time step. The crucial simplification they adopted was to ignore the diffuse, scattered radiation, and to assume that the flow is driven by an ensemble of nonoverlapping, pure-absorption lines. In order to focus on the flow dynamics, they also did not treat a detailed energy balance, but simply assumed that radiative processes would be rapid enough to keep the flow nearly isothermal.

The wind variability simulated by OCR was not exactly intrinsic, but rather was the result of a small-amplitude (1\%), periodic perturbation at the wind base. The strong dependence of driving force on flow speed quickly amplifies this initially small perturbation, giving rise to velocity variations $\Delta v \lesssim 1000 \mathrm{~km} / \mathrm{s}$ by $r \approx 2 R$. An important finding was that these high velocity regions were actually extremely rarefied. This turns out to be 
a very robust result, stemming directly from the linear instability property ( $O$ worki and Rybicki 1984) that wave modes with opposite phase in density and velocity are much more unstable. Although advected away from the star by the supersonic flow, these unstable waves actually propagate inward relative to the fluid, and so as they steepen they naturally evolve into reverse, not forward, shocks. These reverse shocks act to decelerate high-speed, rarefied flow as it impacts slower material that has been compressed into dense shells. This is quite different from the Lucy (1982b) model, in which forward shocks were assumed to abruptly accelerate ambient wind material as it is rammed by a dense, strongly driven flow. In fact, the very low density of this high-speed flow implies that, unlike Lucy's model, only a very small fraction $\left(\sim 10^{-3}\right)$ of the wind material ever undergoes a shock near the maximum amplitude $\Delta v \approx 1000 \mathrm{~km} / \mathrm{s}$. This type of structure is very favorable for explaining several observational features that are difficult to understand in terms of forward shock models (OCR; Owocki 1990), e.g., the hard X-ray tail (Cassinelli and Swank 1983), and the non-sharp, variable blue edges of saturated UV resonance lines (Henrichs, Kaper, and Zwarthoed 1988).

\section{Intrinsic Nature of Wind Variability}

The wind structure computed by OCR arose from amplification of an explicit perturbation introduced at the wind base, but subsequent work (Owocki, Poe, and Castor 1990; Poe, Owocki, and Castor 1990, hereafter POC) has shown that such wind models can also often exhibit an intrinsic variability that persists even in the absence of such explicit perturbations. The incidence of this intrinsic variability was found empirically to depend on the assumed value of $v_{\text {th }} / a$, the ratio of the ion thermal speed to sound speed. For the usual case of driving by CNO ions, an appropriate value of this ratio is $v_{\text {th }} / a \approx 0.3$. However, in order to study the effect of variability arising from explicit perturbations without the complication of a background model that was itself intrinsically variable, OCR found it necessary to assume an artificially high value $v_{\text {th }} / a \gtrsim 1 / 2$. The situation is graphically illustrated in Figs. 1, which show the spatial and temporal variations of velocity and mass loss rate in unperturbed wind models that differ only in the assumed $v_{\text {th }} / a$. In all cases the flow is initially disrupted because the assumed CAK/Sobolev initial condition is not an appropriate steady state for either non-Sobolev model. The model with $v_{\text {th }} / a=0.5$, however, quickly relaxes to a somewhat steeper (POC) steady solution, whereas the models with slightly smaller $v_{\text {th }} / a$ never settle down.

POC showed that the qualitative differences among the variability properties of these cases reflect a difference in the nature of the corresponding steady solutions. Fig. 2 illustrates the steady-state solution topology near the critical (sonic) point. Unlike the usual saddle- or X-type solution that applies, e.g., to the solar wind (Fig. 2a) (Parker 1963) the solution topology in this case is of the nodal type, with not one but two positive slope critical solutions (Fig. 2b). Note that along the steeper slope there is only one distinct solution passing though the critical point, whereas along the shallower slope there is a degenerate family of solutions that pass through this point. POC showed that, for reasonable boundary conditions, the distinct, steeper solution applies when $v_{\text {th }} / a \gtrsim 1 / 2$, whereas the degenerate, shallower solution applies when $v_{\text {th }} / a \leqq 1 / 2$. (These values apply to the simple case of a point star, as was assumed by OCR; subsequent calculations now properly treat a finite stellar disk, and in this case steady, steeper solutions exist at the somewhat lower ratios $v_{\text {th }} / a \gtrsim 3 / 8$.) Apparently, the existence of a well-defined steady solution in the former case is sufficient to enable a time-dependent model without explicit perturbations to relax to this steady state. On the other hand, the lack of a well-defined steady solution for the latter case leads in the time-dependent model to an 


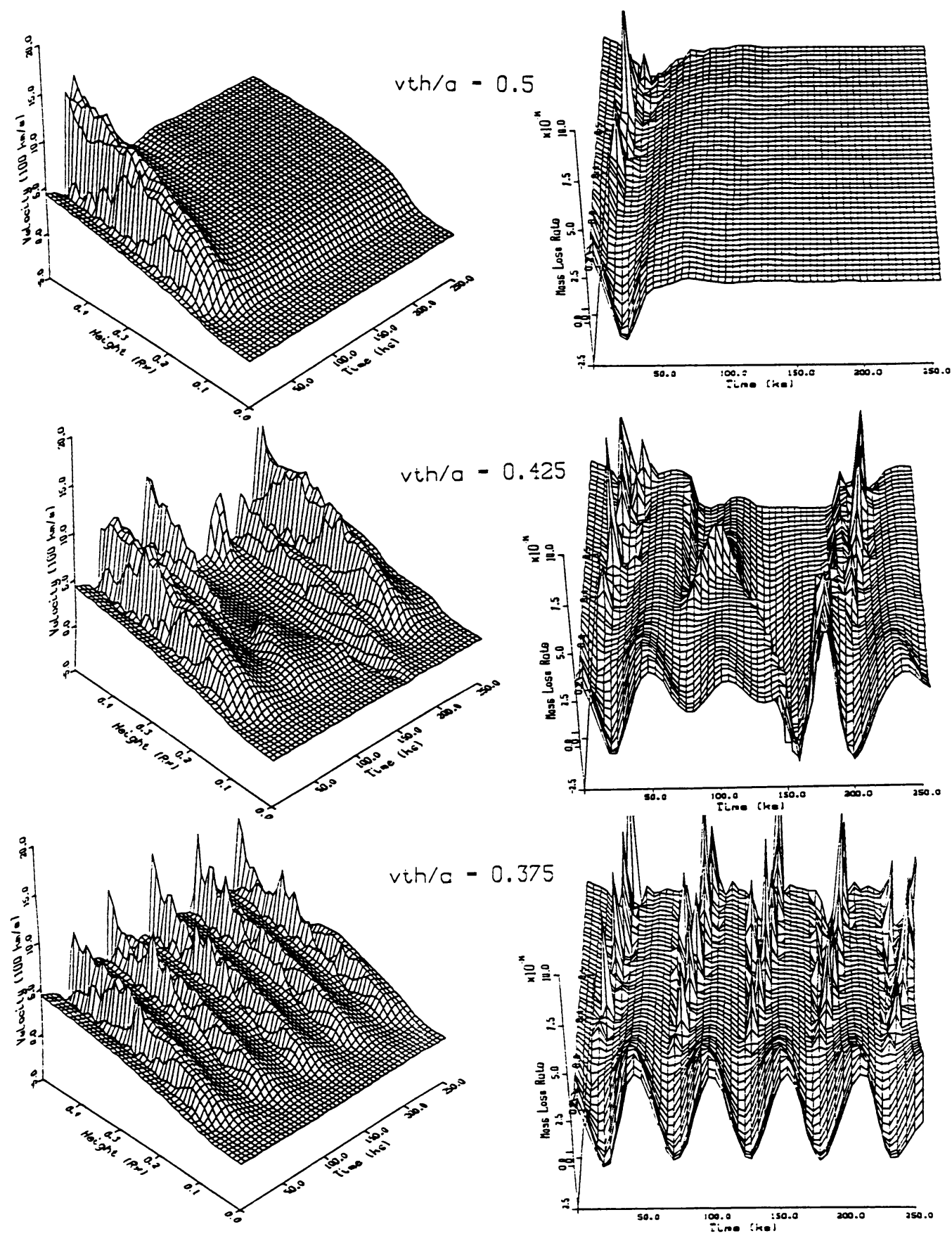

Figure 1: 3-D perspective plots of the height and time variations of velocity (left) and mass loss rate (right) in models with $v_{t h} / a$ of 0.5 (top), 0.425 (middle), and 0.375 (bottom). (For clarity, the mass loss rate is plotted from a perspective looking outward along the height axis.) 
a. Saddle Topology

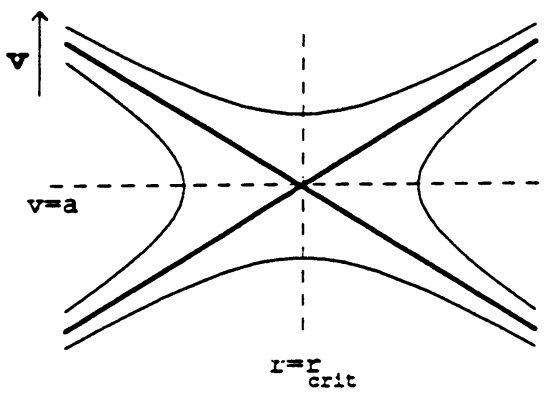

b. Nodal Topology

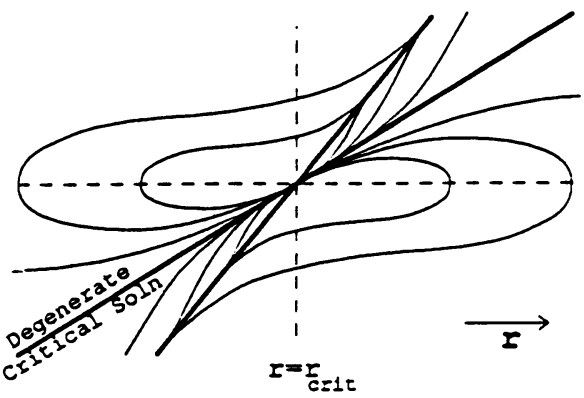

Figure 2: The velocity near the sonic radius for solution topologies of (a) the usual saddle type that applies to the solar wind and (b) a nodal type that applies to absorption-line-driven winds. Note in the nodal case how a large number of solutions converge as they approach the sonic point along the shallower of the two positive critical solutions (heavy solid lines). The implied solution degeneracy leads to the intrinsic variability seen in the lower panels of Fig. 1.

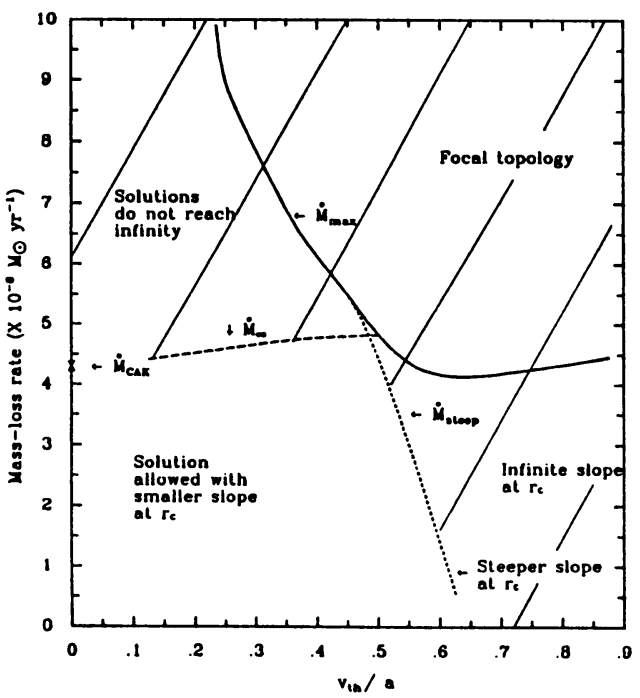

Figure 3: The nature of wind solutions in the parameter plane $\dot{M}$ vs. $v_{t h} / a$. In the hatched region solutions are excluded for various reasons, but flow solutions are possible in the entire non-hatched region.

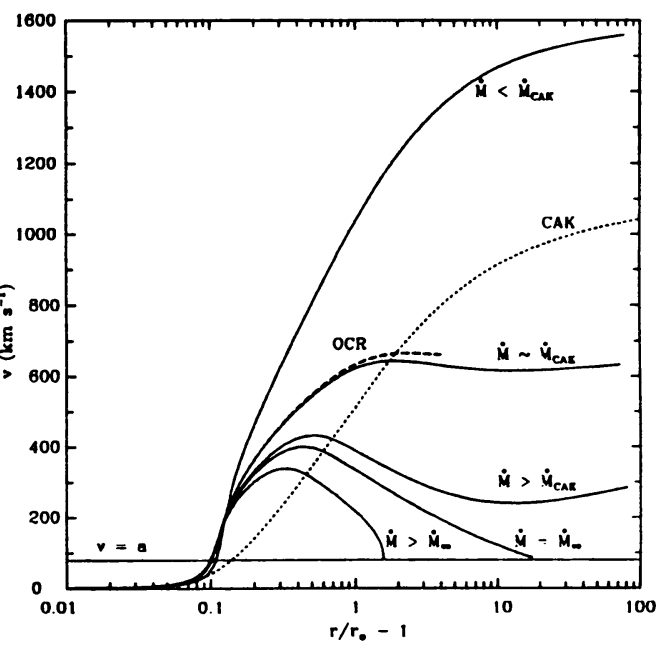

Figure 4: Velocity $v$ vs. radius $r$ for steeper slope solutions with various $\dot{M}$ and $v_{t h} / a$. Note that models with $\dot{M}>\dot{M}_{\infty}$ do not extend to a large radius with a finite speed. 
intrinsic variability in which the flow, roughly speaking, continuously varies among this degenerate family of possible steady solutions.

This kind of solution degeneracy appears to be endemic to line-driving. It was only not apparent in the original CAK model because this model used the Sobolev approximation, even in the subsonic flow, to eliminate all but one "critical" solution. In a more self-consistent model in which one also assumes a vanishing sound speed $a \rightarrow 0$ in the usual Sobolev limit of vanishing thermal speed $v_{\text {th }} \rightarrow 0$, a similar solution degeneracy exists in that the CAK mass loss rate $\dot{M}_{\mathrm{CAK}}$ then only defines an upper limit of the mass loss, not a unique value (POC, Owocki 1990). POC showed that in pure-absorption models in which both $v_{\text {th }}$ and $a$ are nonzero, transonic solutions likewise exist up to a maximum rate $\dot{M}_{\max }$. As shown in Fig. 3, for small thermal speeds $\left(v_{\mathrm{th}} / a \lesssim 0.5\right)$ this can actually exceed $\dot{M}_{\mathrm{CAK}}$, but in this case the CAK rate still roughly corresponds to the maximum rate, $\dot{M}_{\infty}$, that the line force can drive all the way to infinity. Note also that the mass loss rate for the distinct, steeper slope solution, $\dot{M}_{\text {steep }}$, can likewise exceed $\dot{M}_{\infty}$. Fig. 4 then shows that for such steeper solutions with $\dot{M}_{\text {steep }}>\dot{M}_{\infty}$, the velocity reaches a maximum supersonic value and then declines, returning to the sonic speed at a finite "stagnation" radius. Since the steeper solution defines an upper velocity limit for the degenerate family of shallow slope solutions, this means that all solutions with $\dot{M}>\dot{M}_{\infty}$ must likewise stagnate at a finite radius.

These rather peculiar properties of the steady wind solutions actually provide a useful basis for understanding the intrinsic variability seen in the corresponding time-dependent models. For $v_{\text {th }} / a \approx 0.5, \dot{M}_{\max } \approx \dot{M}_{\text {steep }} \approx \dot{M}_{\infty}$, meaning that the distinct, steeper solution through the sonic point has just the mass flux the line-force can drive all the way to infinity; as shown in Figs. $1 \mathrm{a}$ and $1 \mathrm{~b}$, this steeper slope solution thus provides a suitable asymptotic steady-state for the time-dependent model.

Figs. $1 \mathrm{c}$ and $1 \mathrm{~d}$ show, however, that for a somewhat smaller value $v_{\text {th }} / a=0.425$, the flow remains highly variable. The initial base oscillation in mass flux now settles onto a value which the line-force can only lift to a finite height $\approx 0.3 R_{*}$. As matter accumulates at this stagnation point, a shock forms which slows the underlying outflow. By time $t \approx 100$ this turns it into an accretion shock, and until $t \approx 150$, when all the accumulated material falls back onto the star, this completely disrupts the wind. The recovery from this accretion is quite irregular, but ultimately the wind will be reestablished. Soon, however, the mass flux again settles onto a value that is too high to reach infinity, and so another disruption interval of accretion begins. Both the accretion and reestablishment phases of this cycle require on the order of several free-fall times from the stagnation point, and so the entire cycle can take a day or more, depending on the stellar gravity and the height of the stagnation (Section 6).

This stagnation height depends sensitively on $v_{\text {th }} / a$. In models with $v_{\text {th }} / a \approx 0.45$, for example, material actually reaches a height of more than a stellar radius before falling back on the star. On the other hand, for the model with $v_{\text {th }} / a=0.375$, this stagnation height is only slightly above the sonic point itself. In this particular case, the accretion and reestablishment phases turn out to be nicely synchronized and so give rise to the smooth oscillatory behavior seen in Figs. 1e and 1f.

By focusing on the associated cyclical variation in the mass flux (Fig. 1f), we can formulate the following simple picture of the dynamics of this oscillation: First, inward propagation of a small portion of unstable waves that form near the sonic point causes the mass flux in the subsonic flow to temporarily overshoot the continuously sustainable value. As material near the sonic point becomes shadowed from the driving radiation by the enhanced density of the underlying subsonic flow, it decelerates, which then causes the mass flux to undershoot, even becoming briefly inward (the accretion). Once this wave of diminished mass flux reaches the subsonic flow, the overlying material is now less shadowed 
and so accelerates, forming another unstable wave to enhance the mass flux and repeat the cycle.

\section{Characteristic Time Scales}

The time scale for this relatively simple relaxation oscillation is found empirically to scale with the acoustic cutoff period $4 \pi a / g_{*}$. This can be understood physically by noting this is also roughly the sound travel time between the unstable flow just below the sonic point, and the stable subsonic base region below the "thermal" point (i.e., where $v<v_{\mathrm{th}}$ ). In the present models, which for reasons of computational expense (see OCR) assume a sound speed $a=80 \mathrm{~km} / \mathrm{s}$ that is about a factor of 3 too high, this yields a relaxation period of $47,000 \mathrm{~s} \approx 13$ hours. Analogous models with a sound speed $a=25 \mathrm{~km} / \mathrm{s}$ more appropriate to the assumed stellar temperature $(50,000 \mathrm{~K})$ exhibit shorter periods $16,000 \mathrm{~s} \approx 4$ hours. This is about a factor ten longer than the corresponding growth times for small scale instabilities.

However, this is still significantly shorter than the observed repetition time of discrete absorption features (typically $1 / 2-2$ days; Henrichs, Kaper, and Zwarthoed 1988). Despite this disagreement in scale, the time-variations of synthesized absorption profiles do show moving narrow absorption features quite reminiscent of such discrete absorption components (Prinja and Howarth 1988). These arise from outward accelerating dense "shells" whose formation is repeatedly triggered by the quasi-periodic disruptions. For other selected models in which the stagnation height is quite high above the star, the overall time for the semi-regular cycles of accretion/recovery can become correspondingly longer, resulting in profile variations with time-scales approaching that of observed discrete components.

Interestingly, the time scale for the simple relaxation oscillation is roughly comparable to that of optical line-profile variability that has often been described in terms of "nonradial pulsations" (Baade 1988). This might not be just coincidence since, as noted above, this intrinsic wind variability actually does extend down to the subsonic wind base, where these optical lines are formed. Furthermore, the accretion that occurs when the wind stagnates should surely have a substantial impact on the underlying photosphere, perhaps even inducing variations of sufficient magnitude to appear as "pulsations" (Fullerton 1990). Of course, at this point, such a "tail wags dog" scenario of a wind inducing photospheric variability is quite speculative, since these highly simplified, 1-D models ignore several potentially important effects, e.g., stellar rotation.

\section{Role of Scattering}

The lines that drive hot star winds are actually more nearly pure-scattering than pureabsorption, and including the dynamical effect of this diffuse radiation may have important effects on the intrinsic variability. In the supersonic regions of a smooth flow, the near foreaft symmetry of the diffuse radiation field means that the associated net force is typically small; but in the region near and below the sonic point, the radiation escape probability is rapidly increasing, leading to a diffuse field asymmetry. Since the escape probability scales with the velocity gradient, the net asymmetry depends on the second derivative of velocity, and so the effect of the diffuse field can be reasonably modeled as a radiative viscosity. Owocki and Zank (1990) show that such a radiative viscous force can alter or even "break" the solution degeneracy found in pure-absorption models, and this might explain why comoving frame models that include scattering appear to have a well-defined solution (Pauldrach, Puls, and Kudritzki 1986). 
Through the Lucy (1984) line-drag effect (Section 2), scattering might also stabilize the wind base enough to suppress variations that govern the intrinsic variability. The wind instability would then have an advective rather than absolute character, (Bers 1983; Owocki and Rybicki 1986; OPC) for which variability would persist only with some explicit driving from the underlying star, and would only become large amplitude in the supersonic wind. Recently, Owocki and Rybicki (1990) have extended their previous linear stability analyses to the case, relevant to Wolf-Rayet stars, in which the continuum remains optically thick into the supersonic flow. Assuming that the continuum radiation thus attains an angular distribution appropriate to the diffusion approximation, they find that a flow driven by pure-scattering lines is then stabilized by this line-drag effect. They also find, however, that a relatively small amount of photon destruction can cause such a flow region to remain unstable. Determining the linear stability properties of the lower regions of Wolf-Rayet winds will thus require knowing the effective mean destruction probabilities for the lines that drive the wind.

For OB stars, the linear stability properties are relatively well understood, but how scattering effects might influence the nonlinear properties like the intrinsic variability is still not clear. Unfortunately, straightforward calculation of the diffuse field transfer at each time step would be prohibitively expensive, with a timing increase of more than a factor 100 over the present pure-absorption calculation. It will thus be essential to develop less costly approaches. One simple technique currently being tested is based on escape probability methods; this can be implemented with only modest (factor 2) timing increase, and, although quite crude, nonetheless appears capable of incorporating several important effects, like the radiative viscosity and line-drag terms mentioned above. Other methods, such as accelerated Lambda iteration (e.g., Olson, Auer, and Buchler 1986), are also being explored, as is the possibility of some kind of hybrid between the two.

\section{Future Work}

In addition to clarifying the role of scattering in regulating the instability, there is obviously a great deal of work still to be done to include the many other potentially important effects neglected so far, e.g., detailed energy and ionization balance, shock X-ray emission, 2-D or 3-D structure, and stellar rotation. Inclusion of a realistic energy balance in the dynamical wind models is needed to determine the temperature structure and thereby the shock X-ray emission. Likewise, inclusion of ionization balance is needed to determine whether ionization from shock heating and/or X-rays can effect the line-driving and hence the dynamics of the wind and instability. Consideration of rotation and other 2-D (and ultimately 3-D?) effects is needed to determine the likely lateral scales of the dense clumps. For example, can rotation string out such structures in longitude in a manner similar to corotating interaction regions in the solar wind (Mullan 1984, 1985), and thus make it possible for a given dense clump to cover a large enough fraction of the stellar disk to produce the observed discrete absorption components? Finally, perhaps one of the most urgent needs is to develop methods to compare more closely predictions from the theoretical simulations with available observational diagnostics, and thereby guide and test the further theoretical development in this complex but fascinating problem in radiation hydrodynamics.

This work was supported in part by NSF grant AST 88-14580 and NASA grant NAGW1487. Many of the computations were carried out using an allocation of supercomputer time from the San Diego Supercomputer Center. I thank G. Cooper and A. Fullerton for many helpful discussions, questions, and comments. 


\section{References}

Abbott, D. C. 1980, Ap. J., 242, 1183.

Abbott, D. C. 1988, in Solar Wind VI, ed. V. J. Pizzo, T. E. Holzer, and D. G. Sime (Boulder: NCAR/TN-306), p.149.

Abbott, D. C., Bieging, J. H., and Churchwell, E. 1984, Ap. J., 280, 671.

Baade, D. 1988, in O Stars and Wolf-Rayet Stars, ed. P. S. Conti and A. B. Underhill (NASA SP-497), p.137.

Bers, A. 1983, in Handbook of Plasma Physics, Vol. 1: Basic Plasma Physics I, ed. A. A. Galeev and R. N. Sudan (Amsterdam: North-Holland), p. 451.

Carlberg, R. G. 1980, Ap. J., 241, 1131.

Cassinelli, J. P., and Swank, J. H. 1983, Ap. J., $271,681$.

Castor, J. I. 1987, in Instabilities in Luminous Early Type Stars, ed. H. J. G. L. M. Lamers and C. W. H. de Loore, (Dordrecht: Reidel), p. 159.

Castor, J. I., Abbott, D. C., and Klein, R. I. 1975, Ap. J., 195, 157. (CAK)

Fullerton, A. W. 1990, Ph.D. Thesis, University of Toronto.

Harnden, F. R. et al. 1979, Ap. J. (Letters), 234, L51.

Henrichs, H. F., Kaper, L., and Zwarthoed, G. A. A. 1988, in A Decade of UV Astronomy with the IUE Satellite, Vol. 2, ed. by E. J. Rolfe (Paris: ESA), p.145.

Krolik, J. H., and Raymond, J. C. 1985, Ap. J., 298, 660.

Kudritzki, R. P., and Hummer, D. G. 1990, Ann. Rev. Astr. Ap., 28, in press.

Lamers, H. J. G. L. M., and Morton, D. C. 1976, Ap. J. Suppl., 32, 715.

Lucy, L. B. 1982a, Ap. J., 255, 278.

Lucy, L. B. 1982b, Ap. J., 255, 286.

Lucy, L. B. 1984, Ap. J., 284, 351.

Lucy, L. B., and Solomon, P. M. 1970, Ap. J., 159, 879.

MacFarlane, J. J., and Cassinelli, J. P. 1989, Ap. J., 347, 1090.

MacGregor, K. B., Hartmann, L., and Raymond, J. C. 1979, Ap. J., 231, 514.

Mullan, D. J. 1984, Ap. J., 283, 303.

Mullan, D. J. 1985, Astr. Ap., 165, 157.

Olson G. L., Auer, L. H., and Buchler, J. R., J. Q. R. S. T., 35, 431.

Owocki, S. P. 1990, Reviews of Modern Astronomy, 3, (Berlin: Springer), in press.

Owocki, S. P., and Rybicki, G. B. 1984, Ap. J., 284, 337.

Owocki, S. P., and Rybicki, G. B. 1985, Ap. J., 299, 265.

Owocki, S. P., and Rybicki, G. B. 1986, Ap. J., 309, 127.

Owocki, S. P., and Rybicki, G. B. 1990, Ap. J., submitted.

Owocki, S. P., Castor, J. I., and Rybicki, G. B. 1988, Ap. J., 335, 914. (OCR)

Owocki, S. P., Poe, C. H., and Castor, J. I. 1990, in Properties of Hot Luminous Stars, C. D. Garmany, ed. (San Francisco: ASP), p.283.

Owocki, S. P., and Zank, G. P. 1990, Ap. J., submitted.

Parker, E. N. 1963, Interplanetary Dynamical Processes, (New York: Interscience).

Pauldrach, A., Puls, J., and Kudritzki, R. P. 1986, Astr. Ap., 164, 86.

Poe, C. H., Owocki, S. P., and Castor, J. I. 1990, Ap. J., 355, in press. (POC)

Prinja, R. K., and Howarth, I. D. 1988, M. N. R. A. S., 233, 123.

Robert, C., and Moffat, A. F. J. 1990, in Properties of Hot Luminous Stars, C. D. Garmany, ed. (San Francisco: ASP), p.271.

Rybicki, G. B. 1987, in Instabilities in Luminous Early Type Stars, ed. H. J. G. L. M. Lamers and C. W. H. de Loore, (Dordrecht: Reidel), p. 175.

Sobolev, V. V. 1960, Moving Envelopes of Stars, (Cambridge: Harvard University Press).

Willson, L. A., and Hill, S. J. 1979, Ap. J., 228, 854. 


\section{DISCUSSION}

Montmerle: To what extent are the instabilities you find dependent on the fact that you are doing 1-D calculations? Rotation for instance could remove some of the degeneracies? Owocki: I have not yet investigated this question in detail, but I believe going to 2-D and 3-D will mostly add new instabilities, e.g. Rayleigh-Taylor, which will tend to break my "shells" into "clumps". I think it likely that rotation plays a big role in extending the lateral scale of those clumps, helping to make them occult a large enough fraction of the stellar disk to be observable as narrow components. The ultimate structure may be a hybrid of Mullan's CIR picture and this instability model.

Langer: Thinking about a pulsating WR star with a high density (optically thick) wind, would the wind instabilities wipe out the signature of pulsation or would it even amplify it, i.e. would you observe any variations with the period of the pulsation?

Owocki: On the contrary. I think the effect of any underlying pulsation would ultimately be amplified by the wind, and thus be quite observable.

Spurzem: Could you please give a comment on the method used to numerically solve the hydrodynamic equations, e.g. which type of artificial viscosity was utilized?

Owocki: We use van Leer's method of piecewise linear, monotonized advection on a staggered spatial mesh. Quadratic viscosity is used on strong compression to smooth shocks. We tried and abandoned use of linear viscosity on expansion to limit steepness of strong rarefactions. We now limit this by cutting off a few of the very strongest lines from our power-law number distribution of lines vs. opacity. Details can be found in Owocki, Castor and Rybicki (1988).

Sreenivasan: I do not wish to make life any more difficult but I must point out that a realistic energy conservation equation (including rotational effects) must be included to understand really what happens in this case.

Owocki: I agree, and I have a graduate student whose thesis will be to do just that. I should point out, however, that the isothermal assumption is not too unrealistic in the case of dense winds, since then the radiative cooling length behind the shocks can be estimated to be quite small compared to other length scales.

Maeder: What is according to your models the deep physical reason for the difference between the winds (and the mass loss rates) of WR stars and $\mathrm{O}$ stars?

Owocki: Because WR winds are optically thick at the sonic point, the used "core-halo" approximations of $\mathrm{OB}$ winds breaks down, and any radiative driving must be by the diffuse, scattered radiation field. This already means that the wind dynamics and structure must be entirely different than in the OB star case. As for the "deep" reason, I believe it ultimately stems from the lower $\mathrm{H}$-abundance of WR stars. An atmosphere dominated by $\mathrm{He}$ has lower sound speed $a$, and this increases the ratio $v_{t h} / a$ leading to a breakdown of the usual Sobolev approximation near the sonic point. This means the usual direct driving of the core-halo picture fails, and so the diffuse driving must take over. 


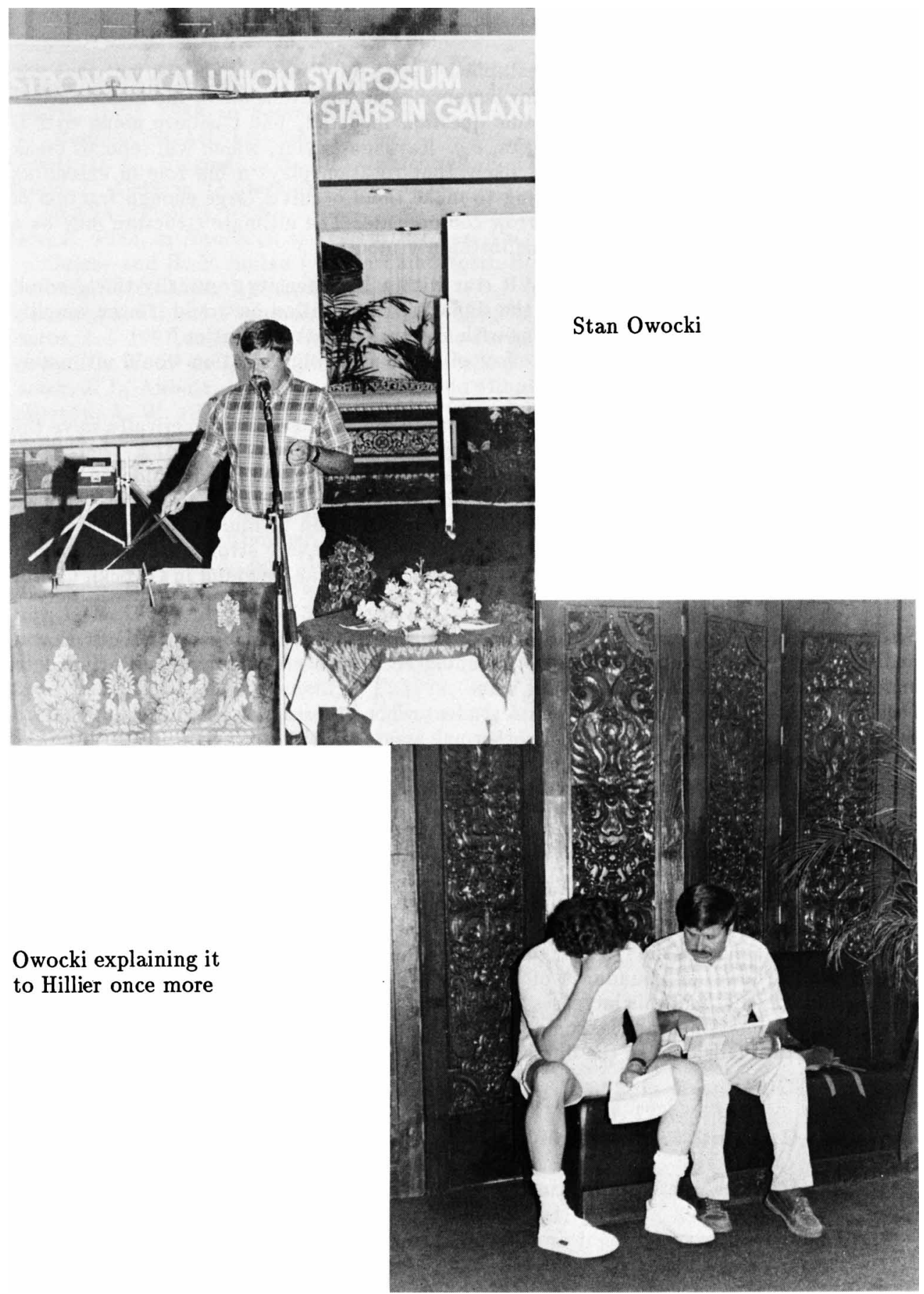

